\title{
Extrinsic extracellular DNA leads to biofilm formation and colocalizes with matrix polysaccharides in the human pathogenic fungus Aspergillus fumigatus
}

\author{
lordana Shopova ${ }^{1,2}$, Sandra Bruns ${ }^{1,2}$, Andreas Thywissen ${ }^{1,2}$, Olaf Kniemeyer ${ }^{1,2,3}$, Axel A. Brakhage ${ }^{1,2 *}$ \\ and Falk Hillmann ${ }^{1,2 *}$
}

${ }^{1}$ Department of Molecular and Applied Microbiology, Leibniz Institute for Natural Product Research and Infection Biology - Hans Knöll Institute, Jena, Germany

2 Department of Microbiology and Molecular Biology, Institute of Microbiology, Friedrich Schiller University, Jena, Germany

${ }^{3}$ Integrated Research and Treatment Center, Center for Sepsis Control and Care, Jena University Hospital, Jena, Germany

\section{Edited by:}

Helio K. Takahashi, Universidade

Federal de Sao Paulo, Brazil

Reviewed by:

Gordon Ramage, University of

Glasgow, UK

Sven Krappmann, University

Hospital Erlangen, Germany

*Correspondence:

Axel A. Brakhage and Falk Hillmann, Department of Molecular and

Applied Microbiology, Leibniz Institute for Natural Product

Research and Infection

Biology - Hans Knöll Institute,

Beutenbergstrasse 11a, 07745 Jena,

Germany.

e-mail: axel.brakhage@hki-jena.de;

falk.hillmann@hki-jena.de
The environmentally acquired fungal pathogen Aspergillus fumigatus causes a variety of severe diseases. Furthermore, it is often found colonizing the respiratory tract of patients suffering from cystic fibrosis. Conidia of this filamentous fungus adhere to substrate surfaces and germinate to form biofilms comprised of dense hyphal networks embedded in an adhesive extracellular matrix (ECM), built predominantly of polysaccharides. These fungal microconsortia are likely to be of clinical relevance, as they have also been observed during growth in the host and they confer drastically reduced susceptibility to antifungals. Little is known about environmental factors or signals contributing to the formation and structural organization of this polysaccharide matrix. Extracellular DNA (eDNA) is an abundant molecule in the mucus-rich surfaces in the lungs of cystic fibrosis patients. Here, we studied its influence on the biofilm establishment and progression of A. fumigatus. Using an in vitro biofilm model eDNA was identified as an efficient biofilm inducer promoting conidial surface adhesion and polysaccharide ECM production. Confocal laser scanning microscopy revealed entirely different ECM architectures depending on the substrates used for biofilm induction. In the presence of serum, adhesive polysaccharides were mainly localized to the hyphal tips appearing as cohesive threads or "halo" areas agglutinating the hyphae. Exogenous DNA altered the structural organization of the biofilm specifically by colocalizing to a grid-like bottom layer of ECM. These results indicate that biofilm formation in A. fumigatus is shaped by certain substrates and in response to host environmental signals.

\section{Keywords: aspergillus, cystic fibrosis, extracellular DNA, extracellular matrix, fungal biofilm}

\section{INTRODUCTION}

Only a few species of the wide spread filamentous fungi are opportunistic pathogens causing often life-threatening diseases in higher animals and humans. Aspergillus fumigatus can be regarded as a model organism of this group, causing primarily pulmonary infections like aspergilloma, cystic fibrosis (CF) associated allergic bronchopulmonary aspergillosis (ABPA) or even often fatal invasive aspergillosis in immunocompromised individuals (Latgé, 2001; Brakhage, 2005; Nivoix et al., 2008). The apparent lack of specific virulence factors has also contributed to the fact that increasing attention is drawn to the infection process and the general life style of this saprophytic mold within the human host (Tekaia and Latgé, 2005). Lung infection propagation is due to conidia of which several hundred are usually inhaled every day. When reaching preformed lung cavities, or the mucus rich surfaces of lung epithelia from CF patients, inhaled conidia can escape the innate immune responses and germinate to form hyphae. Under all growth conditions analyzed so far, hyphae revealed complex multicellular communities characterized by a striking resemblance to biofilms formed by Candida albicans
(Beauvais et al., 2007; Mowat et al., 2009; Loussert et al., 2010). A. fumigatus fungal microconsortia were also observed during surface adherent growth in vitro, conforming to essentially all requirements defined for bacterial biofilms (Mowat et al., 2009; Ramage et al., 2009). Overall, conidia as well as hyphae of A. fumigatus have adhesive and cohesive properties, which are at least in part mediated by an extensive polysaccharide based extracellular matrix (ECM). This ECM covers standard aerially grown colonies but is also present in vivo during host colonization, embedding a highly structured hyphal network, characterized by an increase in resistance to the most prominent antifungal drugs (Beauvais et al., 2007; Mowat et al., 2007; Loussert et al., 2010). In agreement with these data, similar results on ECM production, hyphal organization and resistance to antifungals were also obtained for an extended model demonstrating that $A$. fumigatus biofilms are formed on the surface of human bronchial epithelial and CF bronchial epithelial cells (Seidler et al., 2008).

A. fumigatus biofilms have been subject to several "omics"based studies toward a comprehensive overview of the gene expression on a global scale (Muszkieta et al., 2013). In a first 
approach to understand the molecular basis of biofilm formation in this fungus, we have recently compared global gene and protein expression in planktonic- and biofilm-growing hyphae (Bruns et al., 2010b). A high number of genes involved in primary energy metabolism were found to be highly expressed during early stages of the biofilm covering the first $24 \mathrm{~h}$. Matured biofilms had reduced metabolic activity, but in turn increased production of secondary metabolites. This was most pronounced for gliotoxin-a mycotoxin, whose exact role in virulence is yet to be defined. Recent data suggest the immunosuppressive properties of gliotoxin by targeting downstream molecular pathways involving NF-кB in neutrophils and other phagocytes (Spikes et al., 2008; Scharf et al., 2012). A high-level-expression of secondary metabolite gene clusters was also observed during colony growth of the fungus, revealing striking similarities to growth in biofilms (Gibbons et al., 2012).

The importance of extracellular DNA (eDNA) in biofilms of Gram-positive and Gram-negative bacterial pathogens is widely recognized, but first evidence for its role in inducing and shaping biofilms was given for the opportunistic pathogen Pseudomonas aeruginosa. Here, eDNA triggered the initial establishment of the biofilm, whose structural integrity remained sensitive to DNase I treatment (Whitchurch et al., 2002). The DNA incorporated into the ECM was found to be of chromosomal origin following quorum-sensing-controlled lysis of a bacterial subpopulation (Allesen-Holm et al., 2006). More recent results for $P$. aeruginosa also indicated that eDNA chelates cations resulting in a cellular stress response leading to a drastic increase in antibiotic resistance (Mulcahy et al., 2008). The limited knowledge regarding the function and localization of eDNA during fungal biofilm development originates almost exclusively from results obtained for a few Candida sp. In C. albicans, DNA was not found to be a major constituent of the biofilm. However, treatment with DNase I resulted in the detachment of cells and in an adverse situation, addition of exogenous DNA to mature biofilms led to a significant increase in biomass (Al-Fattani and Douglas, 2006; Martins et al., 2010). Only very recently, it was shown that also in A. fumigatus DNA is released mainly during later stages in biofilm development, conferring a functional role in stability (Rajendran et al., 2013). This DNA release was mediated by autolysis following an increased activity of chitinases and resulted in reduced stress tolerance and lower susceptibility to antifungals. Biomass production in biofilms was also enhanced by DNA from exogenous sources.

Interestingly, free, host-derived DNA is of particular clinical significance contributing to the high viscosity of the mucus in the lungs of CF patients, which is partially counteracted by the administration of nebulized human recombinant DNase I (Shak et al., 1990; Cantin, 1998). CF-sputum DNA mainly originates from necrotic tissues and the release of decondensed chromatin from invading neutrophils undergoing NETosis (Lethem et al., 1990; Papayannopoulos et al., 2011). The formation of neutrophil extracellular traps (NETs) is a part of a suicidal cellular program designed to locally restrict or even kill pathogenic bacteria (Brinkmann et al., 2004; Fuchs et al., 2007). However, NET structures are not only induced by prokaryotes, but were also observed in several in vivo models during pulmonary infection with the most prevalent fungal pathogens $C$. albicans and A. fumigatus (Urban et al., 2009; Bruns et al., 2010a). In contrast to other pathogens, A. fumigatus did not show significantly reduced viability during NETosis (Bruns et al., 2010a). Hence, this study was designed to provide insights into how germination and growth of $A$. fumigatus are affected by the presence of exogenous DNA giving first evidence that it promotes surface adhesion of conidia, biofilm formation and colocalizes with the ECM.

\section{MATERIALS AND METHODS ORGANISMS AND CULTURE CONDITIONS}

All biofilm assays were carried out with conidia harvested from aerial static cultures of $A$. fumigatus CEA17 $\Delta a k u B^{\mathrm{Ku} 80}$ (Da Silva Ferreira et al., 2006), a derivative of the sequenced clinical isolate CEA10 (d'Enfert, 1996). Conidia were harvested from Aspergillus Minimal Medium (AMM) agar plates as previously described (Weidner et al., 1998). Spore suspensions were either freshly harvested or stored for no longer than 1 week at $4^{\circ} \mathrm{C}$ to set up biofilms or to inoculate liquid cultures with RPMI or AMM. Escherichia coli $\mathrm{DH} 5 \alpha$ carrying the high-copy pUC18 plasmid was grown in $500 \mathrm{ml}$ of LB medium with $100 \mu \mathrm{g} / \mathrm{ml}$ of ampicillin and served as a source of plasmid DNA.

\section{BIOFILM GROWTH OF A. fumigatus}

Biofilms of A. fumigatus were set up as described previously by Mowat et al. (2007) and Seidler et al. (2008) with minor modifications. Briefly, spore suspensions were diluted to densities of $1 \times$ $10^{3}-1 \times 10^{6}$ spores in total per well in HEPES-buffered RPMI 1640 with L-glutamine (PAA, Pasching, Austria) and dispensed on flat, presterilized, polystyrene surfaces of 6-well plates or Petri dishes (VWR International bvba, Leuven, Belgium). Several media were tested, e.g., RPMI, RPMI with $10 \%$ (vol/vol) heat inactivated FCS (PAA, Pasching, Austria) and RPMI with DNA from various sources (see below). FCS was previously shown to provide sufficient nutrients to promote growth of a hyphal network and induce biofilm formation (Seidler et al., 2008). Following an initial adherence phase of $4 \mathrm{~h}$ during static incubation in RPMI at $37^{\circ} \mathrm{C}$, unbound conidia were washed three times with $10 \mathrm{ml}$ sterile PBS-Tween 80 solution (Merck, Darmstadt, Germany). Fresh RPMI medium with the indicated additives was added to the adhered conidia and static submerged cultures were grown up to $48 \mathrm{~h}$ at $37^{\circ} \mathrm{C}$.

\section{DNA IN BIOFILM ASSAYS}

If not stated otherwise, commercially available DNA from herring sperm (Sigma-Aldrich, Munich, Germany) was added to RPMI without further purification. This sheared DNA was readily available in larger amounts and is comprised of largely crude oligonucleotides of less than $50 \mathrm{bp}$ in length, as stated in the manufacturer's guidelines. Purity and size were verified by agarose gel electrophoresis as well as spectrophotometric analysis. Other sources of DNA included fungal genomic DNA and plasmid DNA. Fungal genomic DNA was isolated from $200 \mathrm{ml}$ of overnight cultures of A. fumigatus grown in AMM with $1 \%$ $(\mathrm{w} / \mathrm{v})$ of glucose as a carbon source, using standard methods according to Sambrook and Russell (2001). Where indicated, crude DNA preparations were subjected to two successive rounds 
of extraction with equal volumes of phenol/chloroform/isoamyl alcohol (Carl Roth, Karlsruhe, Germany) to remove contaminations of DNA binding proteins such as histones. These DNA preparations contained larger fragments of DNA in the range of 10-30 kbp. Plasmid DNA was isolated from overnight cultures of E. coli $\mathrm{DH} 5 \alpha$ originally transformed with the high-copy pUC18 vector. Large-scale plasmid purification was carried out using the GeneJET plasmid maxiprep Kit (Thermo Fisher Scientific, Dreieich, Germany) according to the manufacturer's instructions. The concentration of DNA added during biofilm development was $1 \mathrm{mg} / \mathrm{ml}$ and this selection was based on previous data obtained for concentrations determined in sputum or BAL fluid of CF patients reaching up to $9.5 \mathrm{mg} / \mathrm{ml}$ (Brandt et al., 1995). Enzymatic digestion of DNA was achieved by treatments with DNase I (Sigma Aldrich, Munich, Germany), which was added at indicated time points of biofilm development to RPMI up to a final concentration of $0.2 \mathrm{mg} / \mathrm{ml}$.

\section{BIOMASS PRODUCTION OF BIOFILMS}

As a measure of fungal biomass production the dry weight of hyphae produced under static and "planktonic" conditions was determined. A. fumigatus was either cultivated in shaken liquid cultures or grown as a biofilm from adherent spores on polystyrene Petri dishes with different media. At indicated time points, mycelia were harvested by scraping from the surface of the Petri dishes or filtration through Miracloth (Merck, Darmstadt, Germany). Both preparations were washed three times with $\mathrm{H}_{2} \mathrm{O}$ and dried up to a constant weight.

\section{ADHESION TEST}

Direct determination of colony forming units (CFUs) is difficult due to the cohesive properties of germinating conidia (Fontaine et al., 2010). Therefore, the conidial adhesion to polystyrene surfaces was quantified directly by visual counting using inverse light microscopy. Conidia were diluted to $2 \times 10^{6}$ spores/well in RPMI alone or with additives and allowed to adhere to the bottom of 6-well plates. At the end of $4 \mathrm{~h}$ of adherence phase, medium was aspirated removing the unbound spores, followed by three sequential washing steps with $10 \mathrm{ml}$ sterile PBS-Tween 80 solution (Merck, Darmstadt, Germany) to remove any remaining unbound spores. For all conditions the number of surface bound conidia in at least 4 fields of defined surface dimension was determined and is expressed as the percentage of total spores in a single assay.

\section{QUANTIFICATION OF EXTRACELLULAR MATRIX}

Polysaccharides of the ECM in fungal biofilms can be readily visualized by the lectin conjugate Concavalin A Alexa-fluor 488 (CAAF, Life Technologies, Darmstadt, Germany) allowing ECM measurements in a semi-quantitative fluorescent microtiter plate assay. CAAF stains primarily $\alpha$-mannopyranosyl and $\alpha$-glucopyranosyl residues in polysaccharides present in the ECM and the fungal cell wall. It was previously employed to stain the ECM of fungal biofilms in CLSM, and also for the quantitative analysis of biofilms (Yang et al., 2006; Chandra et al., 2008; Seidler et al., 2008). For this purpose, biofilms of A. fumigatus were cultivated on black 96-well plates (Brand, Wertheim, Germany) using standardized fresh spore suspensions in $200 \mu \mathrm{l}$ at final concentrations of $10^{4}$ and $10^{5}$ spores per well. Biofilms were grown in static conditions for $48 \mathrm{~h}$ at $37^{\circ} \mathrm{C}$. Fluorescent labelling of the samples was achieved by adding $100 \mu \mathrm{l}$ of $25 \mu \mathrm{g} / \mathrm{ml}$ CAAF in PBS followed by $45 \mathrm{~min}$ incubation at $37^{\circ} \mathrm{C}$ on a plate shaker at $250 \mathrm{rpm}$. Biofilms were then carefully washed three times with sterile PBS to preserve the biofilm architecture and fluorescence intensity was measured on a FluoStar Optima plate reader (BMG LabTechnologies, Ortenberg, Germany) using an excitation filter of $485 \mathrm{~nm}$ and an emission filter of $520 \mathrm{~nm}$. Stock solutions of $5 \mathrm{mg} / \mathrm{ml} \mathrm{CAAF}$ were stored at $-20^{\circ} \mathrm{C}$ and thawed immediately prior use.

\section{BIOFILM VIABILITY TESTING}

The overall viability of fungal biofilms was monitored as an increase in fungal respiration. Similar to the widely used XTT assay (Mowat et al., 2007; Pierce et al., 2008; Seidler et al., 2008), resazurin undergoes a color change upon its metabolic reduction from blue to the pink resorufin and has successfully been used as a respiration indicator in bacterial biofilms (Mariscal et al., 2009). The resazurin assay can be employed either as a fluorimetric or a colorimetric technique. We measured the decrease in absorbance of resazurin at a wavelength of $600 \mathrm{~nm}$. Biofilms were set up in HEPES-buffered RPMI 1640 with L-glutamine (PAA, Cölbe, Germany), lacking the $\mathrm{pH}$ indicator phenol-red, at densities of $10^{4}$ and $10^{5}$ spores per well in flat-bottomed 24-well Nunclon $\Delta$ Multidishes (Thermo Fisher Scientific, Dreieich, Germany). After $4 \mathrm{~h}$ adhesion phase and washing with PBS, new medium was added with resazurin at a final concentration of $88 \mu \mathrm{M}$. A stock solution of resazurin was prepared by filtering through Minisart ${ }^{\circledR}$ syringe filters with a pore size of $0.2 \mu \mathrm{m}$ (Sartorius, Göttingen, Germany) and stored at $4^{\circ} \mathrm{C}$ for no longer than 1 week. Supernatant aliquots of $100 \mu \mathrm{l}$ were transferred to a flat bottom Cellstar ${ }^{\circledR}$ plate (Greiner bio-one, Frickenhausen, Germany) at indicated time intervals and absorbance was measured at $600 \mathrm{~nm}$ on a Tecan Infinite M200 Pro plate reader (Tecan, Männedorf, Switzerland). Data displayed represent the change in resazurin absorbance relative to controls with no cells and unreduced resazurin.

\section{MICROSCOPY IMAGING OF BIOFILMS}

Biofilms for microscopic observation were generated for $24 \mathrm{~h}$ on ibiTreat plastic $35 \mathrm{~mm}$ high $\mu$-dishes (Ibidi $\mathrm{GmbH}$, Munich, Germany) with an initial inoculum density of $10^{4}$ spores in a total volume of $1.0 \mathrm{ml}$ in RPMI supplemented with inducers as mentioned above. After $24 \mathrm{~h}$ of cultivation, biofilms were washed once with sterile PBS and stained for all polysaccharides with $1.0 \mathrm{ml}$ of $25 \mu \mathrm{g} / \mathrm{ml}$ Concanavalin A, Alexa Fluor ${ }^{\circledR} 488$ Conjugate (CAAF, Life Technologies, Darmstadt, Germany) for $45 \mathrm{~min}$ at $37^{\circ} \mathrm{C}$ in static conditions. Unbound CAAF was removed by three gentle washing steps and $1 \mathrm{ml}$ of fresh PBS was added. Chitin structures were stained by adding $2.0 \mu \mathrm{l}$ of $10 \mathrm{mg} / \mathrm{ml}$ of Fluorescent Brightener 28 (Sigma-Aldrich) for further $10 \mathrm{~min}$ at room temperature. Where indicated, samples were also labeled by addition of $5.0 \mu \mathrm{l}$ of $1 \mathrm{mg} / \mathrm{ml}$ of the nucleic acid intercalating dye propidium iodide (PI, Sigma-Aldrich). This dye is membrane impermeable and stains eDNA and damaged or dead hyphae. Without further incubation biofilms were analyzed by 
inverse fluorescence microscopy using an Axiovert 200 M/LSM 5 live confocal laser scanning microscope (CLSM, Carl Zeiss, Jena, Germany). Fluorescence signals were detected using band pass filters of $415-480 \mathrm{~nm}$ (Fluorescent Brightener 28, Life Technologies), 560-675 nm (PI) and 500-525 nm (CAAF). All images were captured and processed using the ZEN 2008 software (Carl Zeiss, Jena, Germany).

\section{STATISTICS AND DATA PRESENTATION}

All data presented represent the means and standard deviations of at least three biological replicates. Graphical presentation and statistical evaluation of the experimental data using the unpaired Students' test ( $t$-test) was carried out with Microsoft Office Excel 2007 software (Microsoft, Redmond, USA). P-values of $<0.05$ were considered as criteria for statistical significance.

\section{RESULTS}

\section{EXTRACELLULAR DNA PROMOTES BIOFILM GROWTH OF A. fumigatus}

RPMI medium contains all nutrients necessary for the growth of A. fumigatus. However, previous work showed that serum additions like $10 \%(\mathrm{v} / \mathrm{v})$ FCS, led to the formation of adhesive biofilms consisting of a dense hyphal network and high amounts of ECM (Seidler et al., 2008; Bruns et al., 2010b). As eDNA was shown to be an abundant molecule in the lung tissues of patients suffering from pulmonary infections, we analyzed how eDNA affects biofilm growth in vitro. A. fumigatus biofilms have been reported to show a specific increase in biofilm biomass in comparison to biomass generated under shaking, planktonic conditions (Seidler et al., 2008). Hence, we determined the dry weight formation during these two growth conditions (Figures 1A,B). In RPMI alone biomass formation was nearly identical when the fungus grew in a planktonic state or as a biofilm. As expected, addition of FCS or eDNA led to increased biomass after $48 \mathrm{~h}$ in both growth forms when compared to RPMI medium alone (Figure 1C). However, only in the presence of eDNA the fungal biomass was specifically increased by at least $20 \%$ when A. fumigatus was cultured as a biofilm. Interestingly, similar biomass production in biofilms was seen for FCS and DNA, despite the macroscopic structures of the two biofilms varied drastically. While biofilms growing with DNA were more irregular and stayed entirely submerged leading to a gel-like appearance, FCS induced the formation of a surface mycelium which did not show any signs of conidia formation (Figure 1B). As a next step, we tracked the time-dependent increase in fungal viability in biofilms as a measure for growth (Figure 2). Fungal respiration due to enhanced metabolic activity results in the concomitant reduction of the redox indicative dye resazurin, which was determined as a decrease in absorbance. Initially conidia were seeded at a concentration of $10^{5}$ per well which was earlier found to be the optimal density for biofilm formation (Mowat et al., 2007). For all conditions metabolic activity was low during a lag period of 16-20 h, which was characterized by the conidial germination and the initial formation of hyphae. In the following $20 \mathrm{~h}$ the formation of mycelial monolayers was followed by rapid growth with nearly linear kinetics. In RPMI alone fungal cells reduced resazurin at a rate of $0.011 \mathrm{~h}^{-1}$, which rose to $0.017 \mathrm{~h}^{-1}$ with FCS (Figure 2, inset). Growth kinetics with eDNA were exceeding those obtained for FCS and were more than
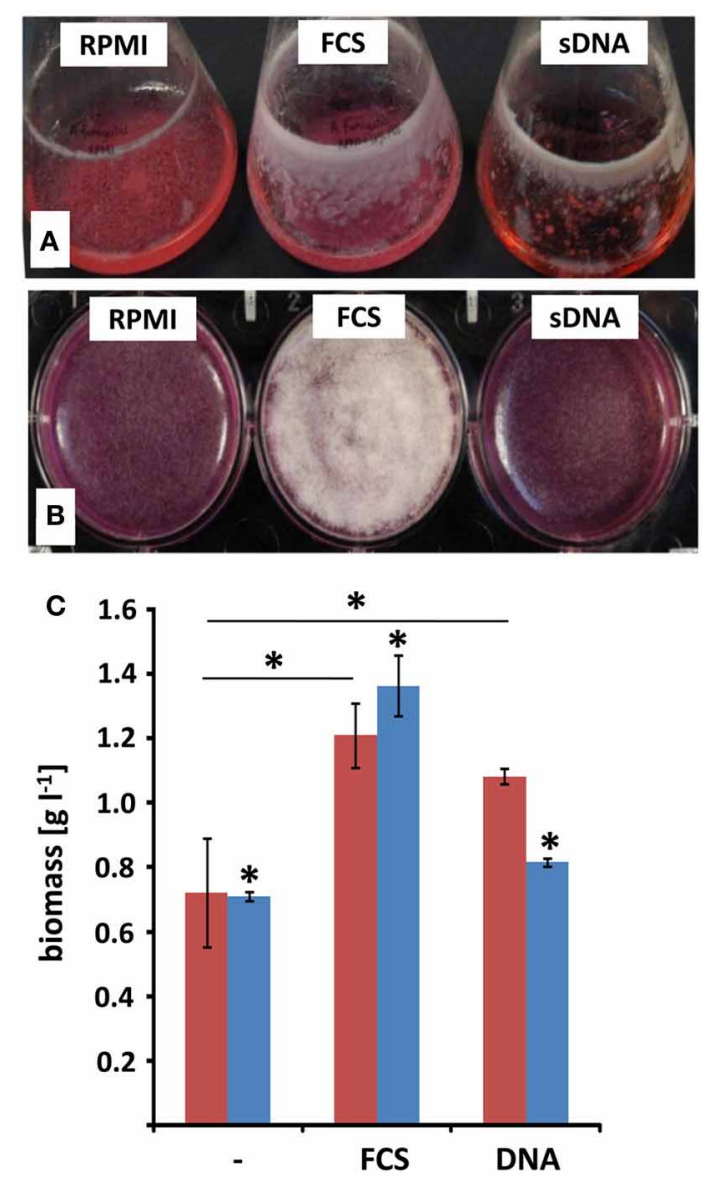

FIGURE 1 | Surface adhesion and biofilm formation with extrinsic DNA. A. fumigatus conidia were grown either in RPMI without additives (RPMI), with addition of $10 \%[\mathrm{v} / \mathrm{v}]$ fetal calf serum (FCS) or with $1 \mathrm{mg} \mathrm{ml}^{-1}$ sheared herring sperm DNA (sDNA) in submerged, shaken culture ( $\mathbf{A}$ ) or in static biofilm inducing conditions (B). Growth was monitored after $48 \mathrm{~h}$ and expressed as total biomass formation during shaking (blue) and static (red) culture conditions (C). Displayed values represent the means and SDs of three independent experiments and asterisks indicate $p<0.05$ in Student's t-test.

two fold higher when compared to RPMI alone, leading to a maximal reduction rate of $0.025 \mathrm{~h}^{-1}$. Similar kinetics were also reproducibly obtained when starting from a lower conidia inoculum of $10^{4}$ per well (not shown) further supporting the hypothesis that extrinsic DNA acts as an efficient inducer of biofilm growth.

\section{EXTRINSIC DNA INDUCES CONIDIAL SURFACE ADHESION}

For bacteria, and especially for the opportunistic pathogen $P$. aeruginosa, it has been well-established that eDNA promotes biofilm formation already at the earliest stage of development (Whitchurch et al., 2002). Therefore, we analyzed whether the presence of DNA also influenced the initial phases of biofilm formation in A. fumigatus. For single cell organisms like bacteria, cellular adhesion is often quantified by counting the CFUs. This standard test proved to be difficult and produced unreliable data sets, presumably due to the extensive aggregation of fungal 


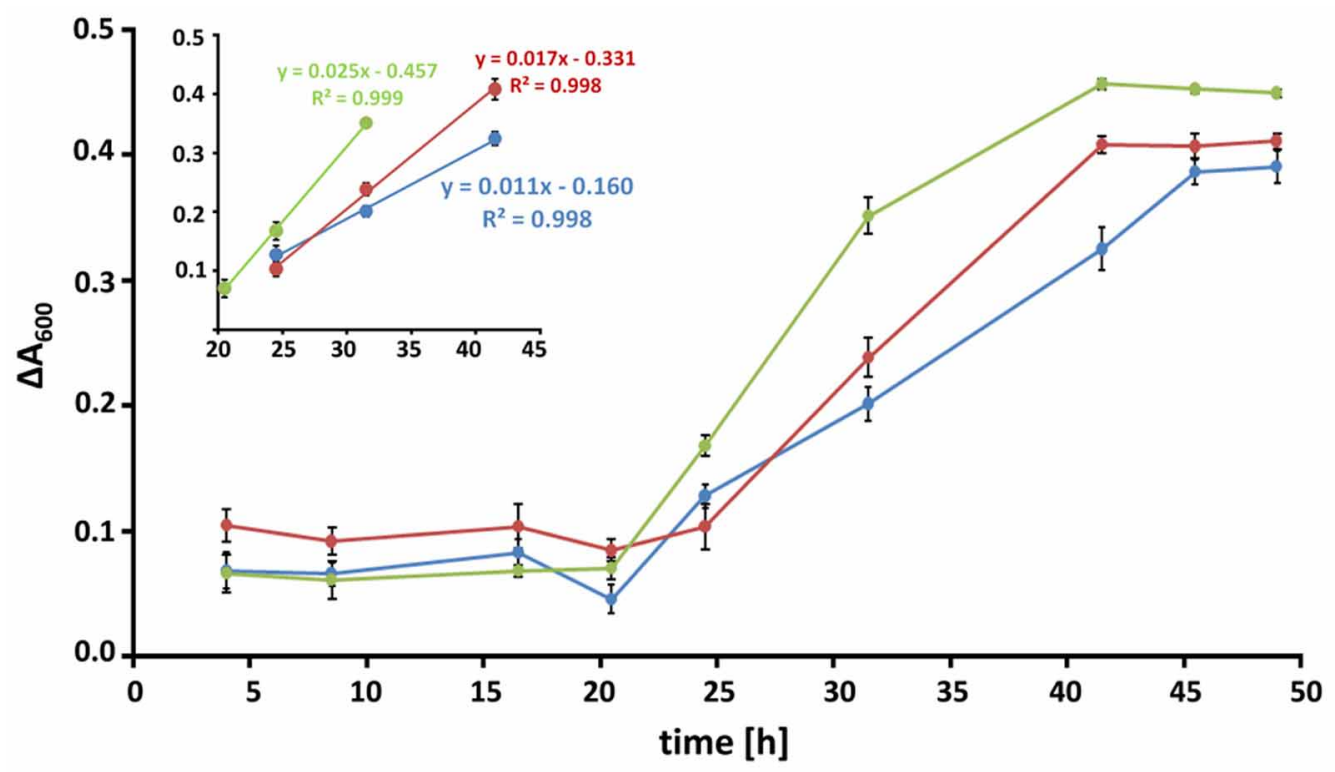

FIGURE 2 | Kinetics of biofilm formation. Biofilms at conidial densities of $10^{5}$ per well were grown in RPMI alone (blue), in the presence of $10 \%[\mathrm{v} / \mathrm{v}]$ FCS (red) or $1 \mathrm{mg} / \mathrm{ml}$ of DNA (green) and fungal viability was monitored over time. Metabolic reduction of resazurin was measured as the decrease in absorbance at a wavelength of $600 \mathrm{~nm}$. Data are expressed as the change in absorbance relative to an untreated control and represent the means and SDs of a biological triplicate. Time periods of linear growth kinetics for all three conditions are displayed in the inset. conidia often observed in liquid (Fontaine et al., 2010). As a consequence, we quantified surface adherent conidia directly by microscopic counting following a $4 \mathrm{~h}$ initial adherence phase (Figures 3A,B). This experimental approach revealed significant differences in conidial adhesion depending on the presence of FCS or DNA. The addition of any of the two components also did not alter germination, as imaging after $8 \mathrm{~h}$ indicated that both rate and frequency stayed essentially constant under all three conditions. In RPMI alone, after $4 \mathrm{~h}$ only few conidia had adhered to the surface of the well, but the presence of FCS or DNA increased adhesion by at least a factor of two. Addition of DNase I revealed that this process was indeed specific to DNA, as the treatment at early time points drastically reduced the ability of the conidia for surface adhesion down to RPMI comparable levels.

\section{EXTRINSIC DNA LEADS TO PRODUCTION OF MATRIX POLYSACCHARIDES}

Surface adhesive growth of $A$. fumigatus was promoted by the presence of DNA and dry weight measurements demonstrated an increased biomass production under these conditions. As a follow-up of this study we addressed the question whether increased viability also corresponds to enhanced formation of cellular polysaccharides. Concanavalin A coupled to fluorescent Alexa-fluor 488 (CAAF) specifically stains polysaccharides of the ECM and the fungal cell wall. Here, we also included DNA with different purities and from various sources and measured total fungal polysaccharides in mature biofilms after $48 \mathrm{~h}$ (Figure 4A). As expected, polysaccharide production with FCS or DNA was higher than in RPMI alone. When polysaccharide measurements were normalized to biomass, these values indicated that matrix formation was specifically enhanced by more than a factor of two in the presence of FCS and DNA (Figure 4B). DNA from different sources all enhanced polysaccharide production to different extent. Interestingly, ECM formation was also dependent on the length of the DNA fragments. The two DNA preparations with shorter DNA fragments $(3.0 \mathrm{kbp}$ for plasmid and $\sim 0.05 \mathrm{kbp}$ for sheared DNA) led to higher induction when compared to crude isolations of fungal genomic DNA ( $>30 \mathrm{kbp}$ ). Purity of the DNA was a minor factor as multiple phenol chloroform extractions of the genomic DNA had no significant influence on ECM production (Figure 4A).

\section{STRUCTURE OF BIOFILMS FORMED IN THE PRESENCE OF EDNA}

Biofilm structures of fungi can vary greatly with regard to surfaces, medium composition or even conidial seeding density. In A. fumigatus such variations are at least partially reflected by differences in the production of ECM. Hyphae found in aspergilloma are covered by an adhesive, polysaccharide-based ECM, providing structure and stability to the hyphal network (Loussert et al., 2010). Similar observations were made earlier for aerially grown colonies of the fungus or hyphae growing submerged under biofilm-inducing conditions (Beauvais et al., 2007; Mowat et al., 2007; Seidler et al., 2008). As eDNA revealed to be an efficient inducer of biofilm, we analyzed whether this also affected the structure of these biofilms. As mature biofilms were characterized by a massive formation of hyphal structures, leading to a thick network, which prevented the visualization of detailed structures, we focused on the end of the initial maturation phase after $24 \mathrm{~h}$. At this time point CLSM imaging could still reveal detailed structural information on the developing complex three-dimensional networks originating from a single germinating conidium (Figure 5). CAAF staining indicated 


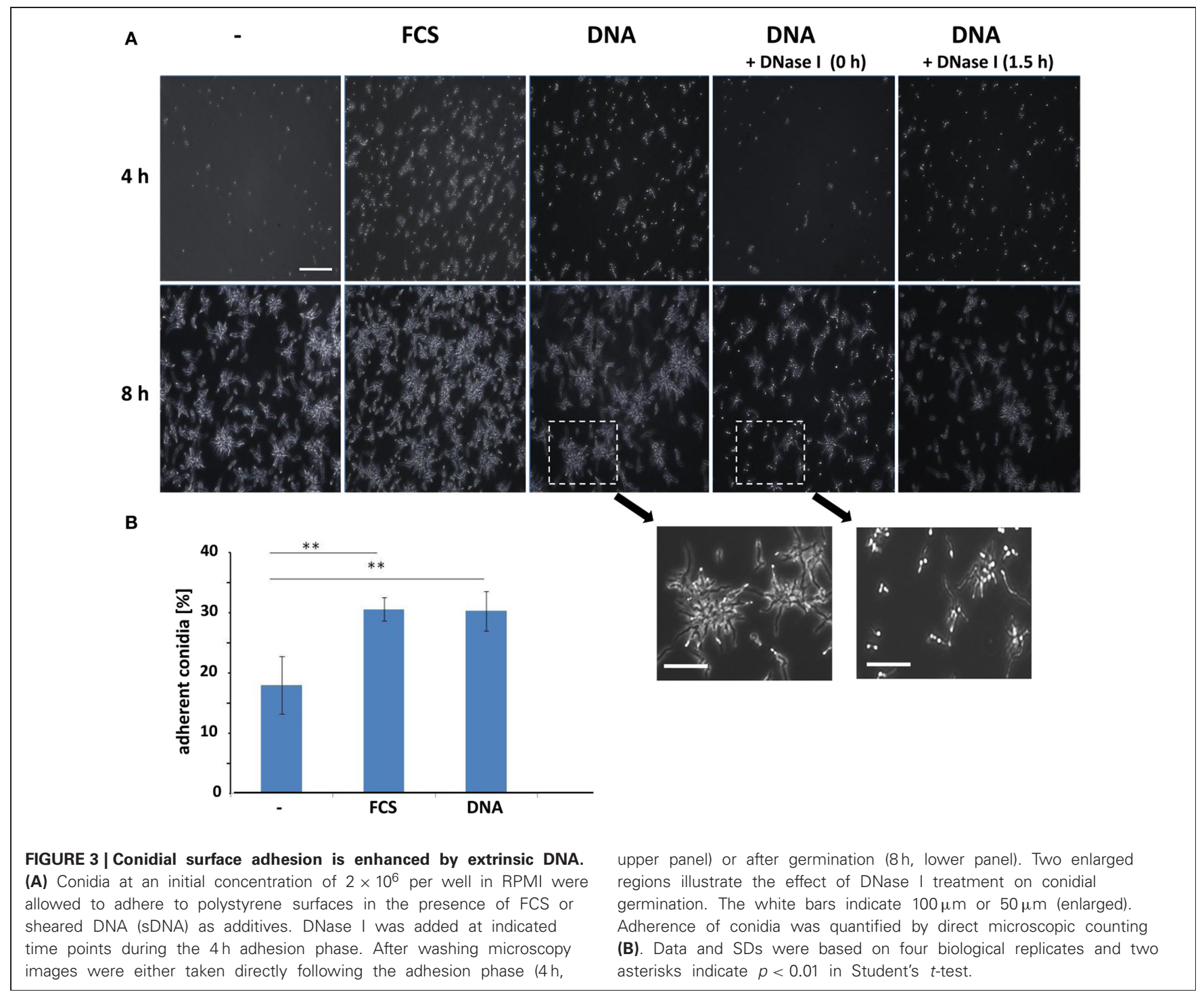

that RPMI-grown fungal populations were composed of weakly attached hyphae lacking ECM polysaccharides, and thus, showed essentially no biofilm formation. In FCS, hyphae showed augmented branching with a diameter in the range of $3.5-4.5 \mu \mathrm{m}$, a size considerably larger in comparison with hyphae grown in RPMI alone $(2.2-3.5 \mu \mathrm{m})$. Positive CAAF signals in FCS samples clearly showed that the distal hyphal tips were the predominant site of ECM localization and the exopolymeric material appeared as a cloudy "halo" around the tips of the hyphae. In agreement with earlier observations (Beauvais et al., 2007), the cohesive properties of the ECM under these conditions was also apparent, showing that these polysaccharides not only yield a protective cover, but also form sticky threads connecting the hyphal tips. The presence of DNA had only a minor influence on the thickness of the hyphae with average diameters ranging from 2.9 to $3.2 \mu \mathrm{m}$. In accordance with a smaller hyphal diameter seen in the presence of eDNA, relatively small amounts of the ECM were observed around the hyphae or at their tips. Instead, under these conditions the ECM was widely distributed between the hyphae forming a loose network of polysaccharides within the biofilm.

Only in the presence of eDNA, the entire hyphal network appeared to be embedded in a diffusely localized ECM, similar as earlier described for mature biofilms (Seidler et al., 2008). This was even more pronounced when imaging the distal hyphae of the biofilms (Figure 6).

\section{EXTRINSIC DNA COLOCALIZES WITH THE EXTRACELLULAR MATRIX}

In the polymeric matrices of a wide range of biofilms eDNA represents an important structural component (Martins et al., 2012; Rajendran et al., 2013). As we observed that DNA from extrinsic sources also induced and shaped biofilm formation, we addressed the question whether exogenous eDNA would also become incorporated into the ECM of A. fumigatus. Fluorescence staining of DNA with PI was previously shown to be a useful tool for DNA staining following membrane disruptions of bacteria, but also for the visualization of DNA structures and their localization during the formation of extracellular traps by 

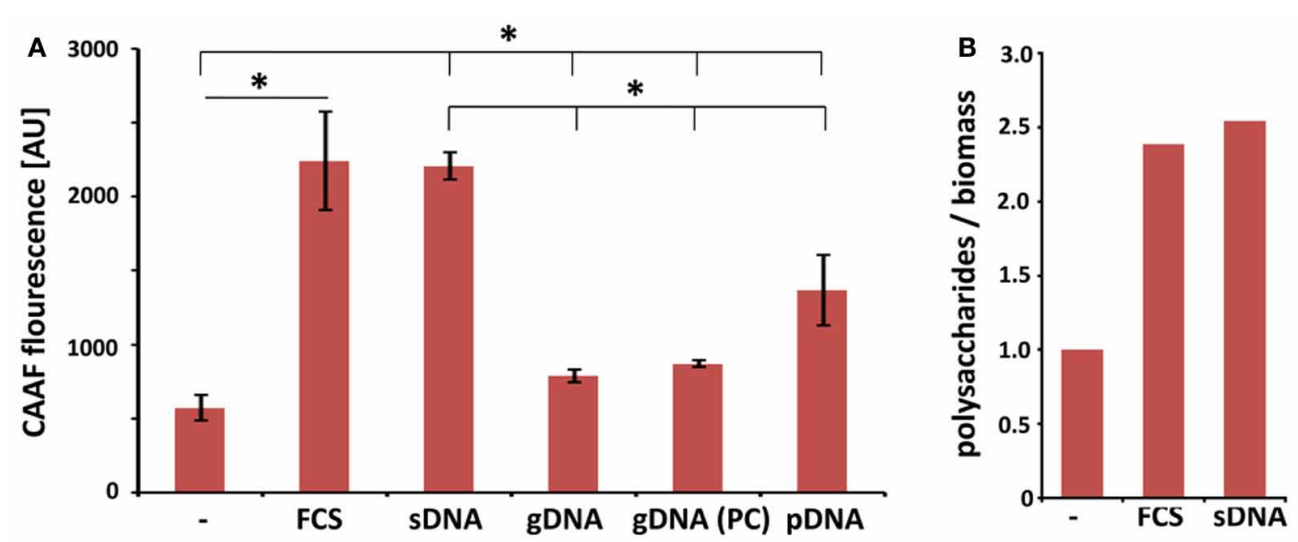

FIGURE 4 | Extrinsic DNA increases ECM formation in biofilms.

(A) CAAF fluorescence of total fungal polysaccharides was determined in biofilms seeded at an initial density of $10^{4}$ per well and used as a measure for ECM production in biofilms in the presence of $1 \mathrm{mg}$ $\mathrm{ml}^{-1}$ DNA from indicated sources (sDNA, sheared herring sperm DNA; gDNA, genomic DNA from A. fumigatus; PC, phenol chloroform extracted DNA; pDNA; purified plasmid DNA). Displayed values represent the means and SDs of three independent experiments and an asterisks indicate $p<0.05$ in an unpaired $t$-test. (B) FCS- and DNA-specific increases in total polysaccharide production are expressed as the ratio of CAAF fluorescence to biomass normalized to untreated controls.

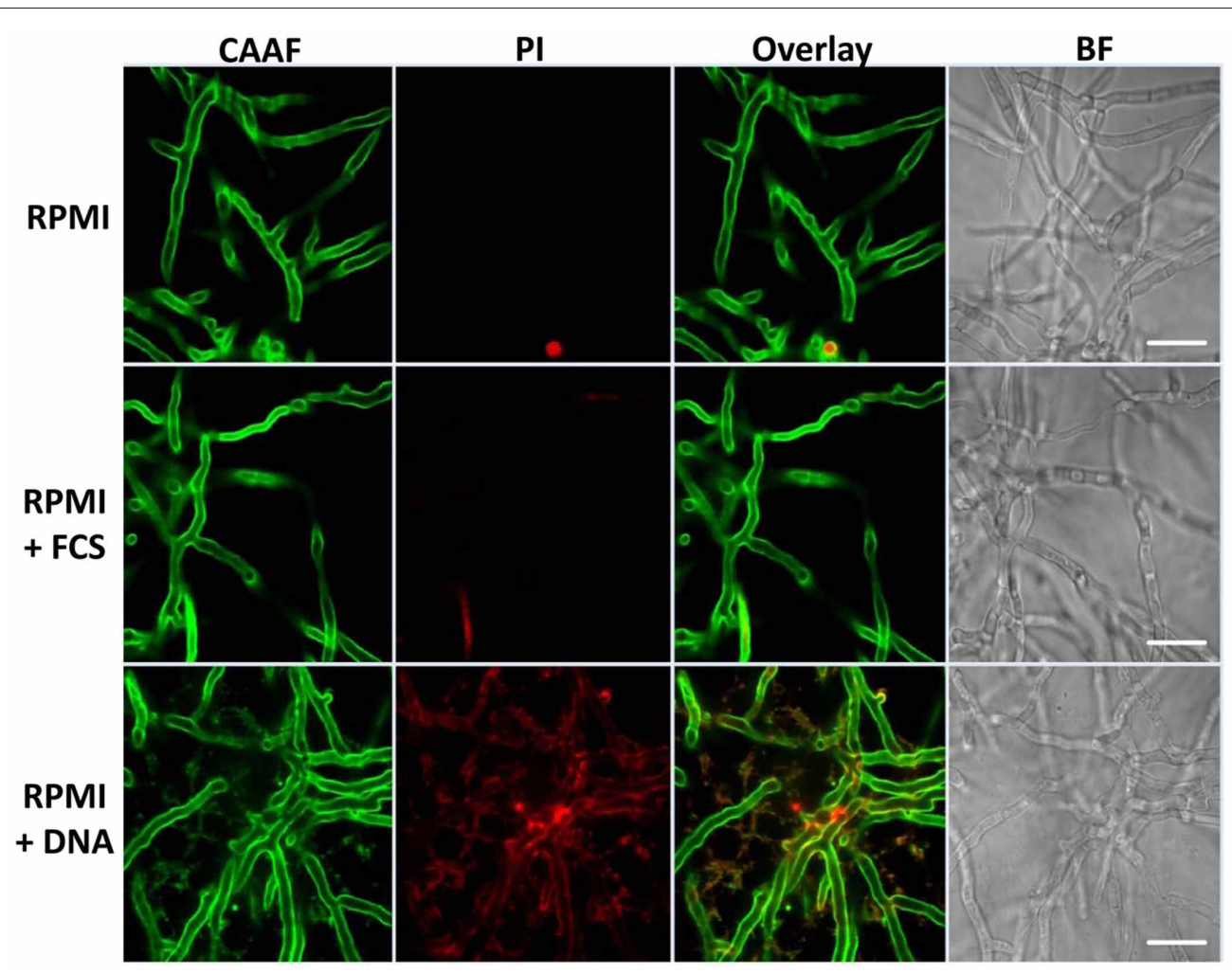

FIGURE 5 | Extrinsic DNA localizes to cellular polysaccharides. Biofilms were grown for $24 \mathrm{~h}$ in RPMI with $10 \%$ [v/v] FCS and $1 \mathrm{mg}$ $\mathrm{ml}^{-1}$ sheared DNA and stained for total polysaccharides using Concavalin A coupled to fluorescent Alexa-fluor 488 (CAAF) or for nucleic acid using propidium iodide (PI). The two right panels represent an overlay of CLSM images from the CAAF and PI channel and the according brightfield images (BF), respectively. White bars indicate $20 \mu \mathrm{m}$. human neutrophils. Young biofilms grown in the absence of eDNA showed only some residual staining when treated with this membrane impermeable, nucleic-acid-intercalating dye. Positive signals in DNA-free biofilms were restricted only to membrane disrupted fungal cells, as is expected for the origin of conidial germination and for a few dead hyphae (Figure 6). This finding further proved that PI was DNA specific and is in agreement with a recent study, showing that only mature biofilms release 


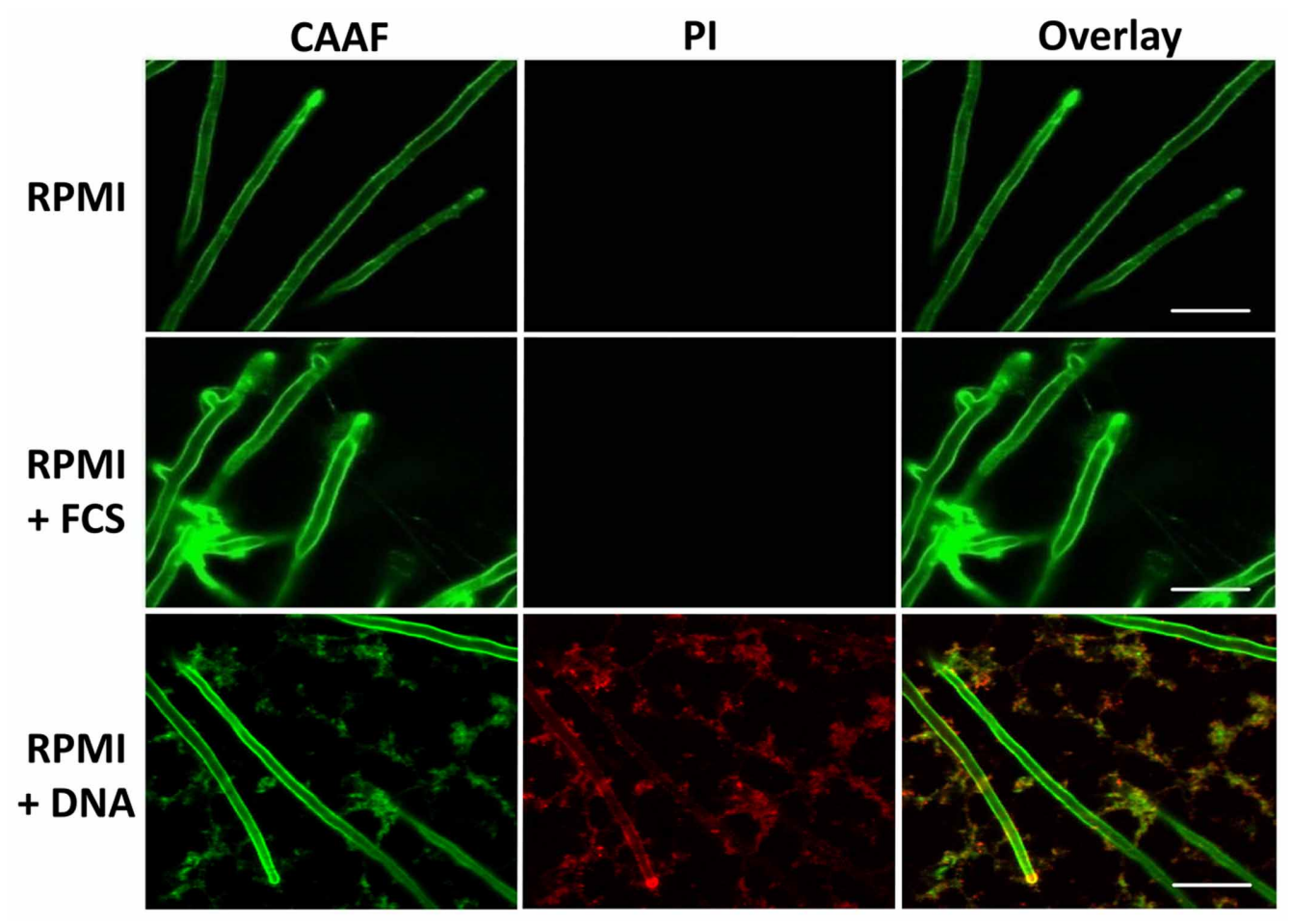

FIGURE 6|Extrinsic DNA influences ECM distribution. Peripheries of biofilms grown with RPMI with either FCS or sDNA were imaged by CLSM displaying the tips of terminal hyphae. Note the cohesive properties of ECM polysaccharides in the presence of FCS and the network-like distribution of the ECM in response to DNA. White bars indicate $20 \mu \mathrm{m}$. significant amounts of genomic DNA by autolysis (Rajendran et al., 2013). When biofilms were formed in the presence of eDNA, PI showed an identical signal distribution in the biofilm as seen for the extracellular polysaccharide-specific CAAF signal (Figure 6). To check whether these signals were specific and the DNA did indeed colocalize with the ECM, we used DNA of various lengths and sources. Only when DNA was subjected to DNase I digestion, PI staining of the biofilm was completely diminished (Figure 7). However, DNase I treatment did not promote any obvious structural changes at this stage of biofilm developement, as the overall three-dimensional distribution of polysaccharide in the hyphal network was not altered (Figures 7B,C). Furthermore, DNA bound to polysaccharide was mainly observed as a bedding layer of the biofilm, providing additional evidence for its functional role during the first stages of development.

\section{DISCUSSION}

Only in the past years increasing evidence has favored the idea that the hyphal network of filamentous fungi resembles biofilm structures. Up to now, however, there is only little knowledge about how filamentous growth contributes to the virulence of A. fumigatus during the different disease patterns caused by this opportunistic pathogen. During CF A. fumigatus is a common lung colonizer most likely favored by chronic antibiotic therapy in older patients (Pihet et al., 2009). The mucus-rich environment is characterized by high concentrations of free DNA originating from a subpopulation of attracted neutrophils undergoing
NETosis. Using an in vitro model for biofilm development, we showed that eDNA supports the surface adhesion of fungal spores, and that the free DNA becomes part of the ECM surrounding the fungal biofilm cells.

Filamentous fungi such as A. fumigatus grow well under submerged shaking conditions in minimal medium, but first investigations demonstrated that addition of complex nutrient sources like FCS supports surface attachment and subsequent biofilm formation. Fetuin A was identified as one crucial serum component involved in this process (Toyotome et al., 2012), raising the question to which extent biofilm formation is favored by specific components present in FCS or complex nutritional environments. When we quantified total fungal dry mass produced under the different growth conditions, the nutrient-rich FCS resulted in an increase in biomass as expected. However, a biofilm-specific increase in dry weight was only seen upon addition of exogenous DNA, leading to the conclusion that this additive supported biomass formation primarily during static growth conditions. Enhanced biomass formation in the presence of FCS and DNA was also reflected by the increased viability in biofilms, measured as an increased reduction of resazurin to resofurin. It seemed likely that this specific increase in viability would also result in higher production of polysaccharides as part of the ECM, as also observed in prior studies on $A$. fumigatus biofilms (Seidler et al., 2008). For a detailed analysis of polysaccharide production during biofilm formation we employed a semi-quantitative assay based on CAAF fluorescence 


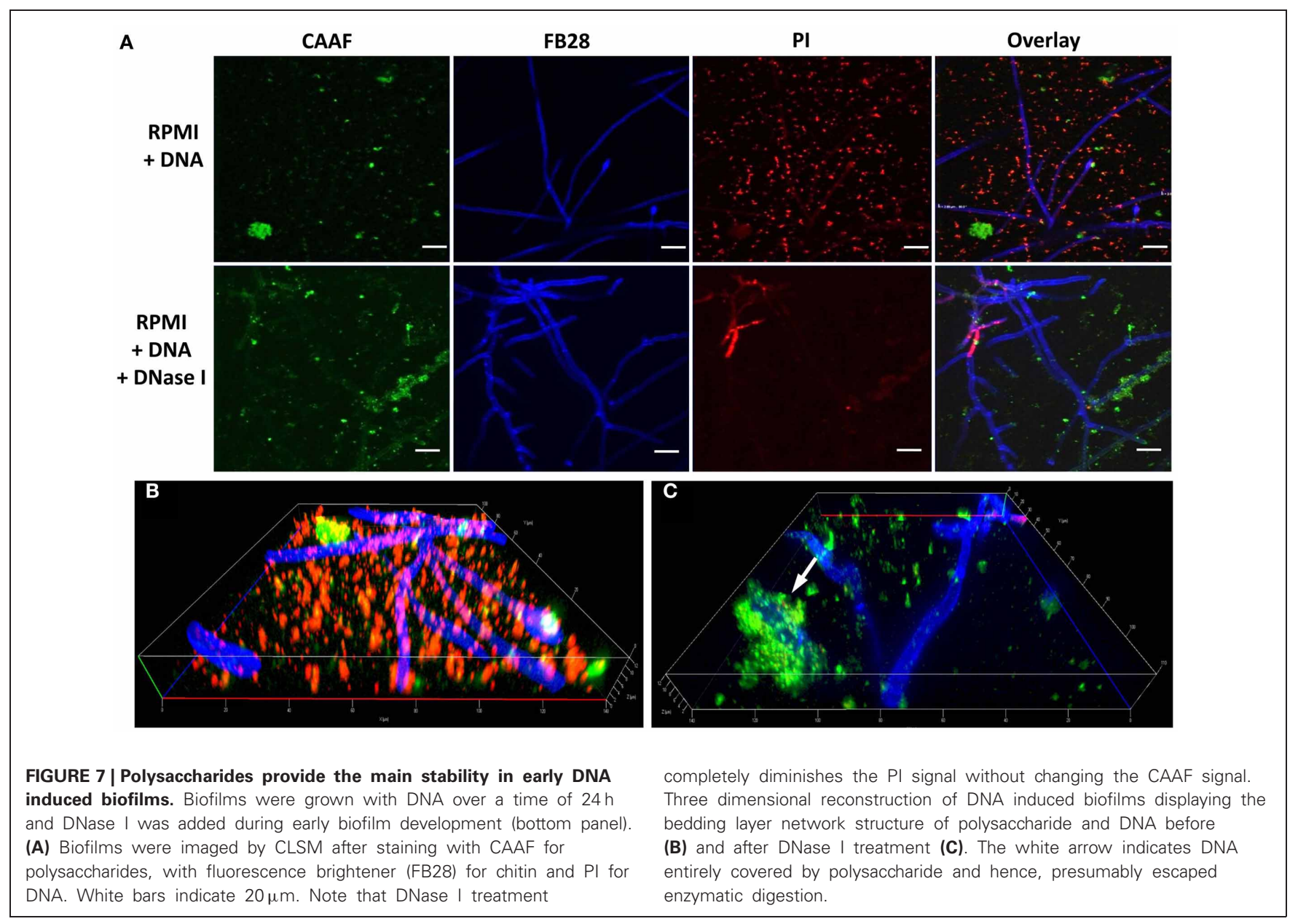

measurements in microtiter plates. Similar amounts of extracellular polysaccharides were produced when FCS was substituted by DNA, and correlated to the cellular viability under the same conditions. Also, when normalized to the biomass production, it became obvious that polysaccharide production was specifically induced in the fungus. Interestingly, polysaccharide production seemed to correlate negatively to the lengths of the DNA fragments, suggesting rather an electrostatic interaction than any type of structural recognition. For a further support we studied closer the initial phase of biofilm formation. Conidial surface adhesion was supported by eDNA to almost the same extent as FCS. It is interesting to note that nearly all adhered spores revealed synchronized germination, as judged by light microscopy during the initial phases of biofilm development following the $4 \mathrm{~h}$ adherence phase. DNase I treatments throughout the adherence phase reduced the number of adherent spores, indicating the importance of DNA during adhesion. This is in perfect agreement with a recent study showing that DNase I treatment drastically disturbed the structural integrity of mature biofilms of A. fumigatus (Rajendran et al., 2013).

As a next step we studied whether eDNA had any influence on the structure and organization of the hyphal network. CLSM revealed that with FCS the ECM polysaccharides were primarily localized at the hyphal tips, thereby specifically enclosing individual hyphae during linear growth and connecting distant hyphae via cohesive threads. Previous work showed that biofilms comprise a dense hyphal network embedded in a cohesive polysaccharide matrix and similar structures were also observed for hyphae recovered from the lungs of aspergillosis patients, and even more pronounced for hyphae during aspergilloma (Beauvais et al., 2007; Seidler et al., 2008; Loussert et al., 2010). In the presence of DNA a smaller diameter of the hyphae was accompanied by a wide spread ECM that was shaped like a network, resembling the situation during static aerial colony formation (Beauvais et al., 2007). DNA colocalized with the polysaccharide network as revealed by the specific DNase I sensitive PI staining of the ECM. The electrostatic affinity of nucleic acids for polysaccharides of $A$. fumigatus was addressed previously, when hyphae were found to efficiently scavenge small RNAs and DNA oligonucleotides within their cell wall (Jöchl et al., 2009). It is therefore conceivable that the presence of free DNA might trigger enhanced polysaccharide synthesis, resulting in increased adhesion and ECM formation. Furthermore, DNA as a part of the ECM was primarily detected on the bottom, bedding layer of the biofilms, similar as seen earlier for young biofilms of $P$. aeruginosa, where eDNA formed grid-like structures on the substratum (Allesen-Holm et al., 2006). Also, for a number of other bacteria, DNA was shown to be a crucial component of the biofilm 
(see Flemming and Wingender, 2010 for review). In two recent studies this was also demonstrated for the fungal species C. albicans and A. fumigatus (Martins et al., 2012; Rajendran et al., 2013). For the latter one, structural support by autolysis-derived DNA is of major importance during later stages of biofilm development and was also proposed to be of medical importance due to reduced susceptibility to antifungals, which was partially counteracted by the concomitant addition of DNase (Rajendran et al., 2013). In agreement with this finding, we found that extrinsic DNA enhanced surface adhesion of conidia and shaped the organization and distribution of the ECM during early biofilm

\section{REFERENCES}

Al-Fattani, M. A., and Douglas, L. J. (2006). Biofilm matrix of Candida albicans and Candida tropicalis: chemical composition and role in drug resistance. J. Med. Microbiol. 55, 999-1008. doi: 10.1099/jmm.0. 46569-0

Allesen-Holm, M., Barken, K. B., Yang, L., Klausen, M., Webb, J. S., Kjelleberg, S., et al. (2006). A characterization of DNA release in Pseudomonas aeruginosa cultures and biofilms. Mol. Microbiol. 59, 1114-1128. doi: 10.1111/j.13652958.2005.05008

Beauvais, A., Schmidt, C., Guadagnini, S., Roux, P., Perret, E., Henry, C., et al. (2007). An extracellular matrix glues together the aerial-grown hyphae of Aspergillus fumigatus. Cell. Microbiol. 9, 1588-1600. doi: $\quad 10.1111 / j .1462-5822.2007$. 00895

Brakhage, A. A. (2005). Systemic fungal infections caused by Aspergillus species: epidemiology, infection process and virulence determinants. Curr. Drug Targets 6, 875-886. doi: 10.2174/138945005774912717

Brandt, T., Breitenstein, S., von der Hardt, H., and Tümmler, B. (1995). DNA concentration and length in sputum of patients with cystic fibrosis during inhalation with recombinant human DNase. Thorax 50, 880-882.

Brinkmann, V., Reichard, U., Goosmann, C., Fauler, B., Uhlemann, Y., Weiss, D. S., et al. (2004). Neutrophil extracellular traps kill bacteria. Science 303, 1532-1535. doi: 10.1126/science. 1092385

Bruns, S., Kniemeyer, O., Hasenberg, M., Aimanianda, V., Nietzsche, S., Thywissen, A., et al. (2010a). Production of extracellular traps against Aspergillus fumigatus in vitro and in infected lung tissue is dependent on invading neutrophils and influenced by hydrophobin RodA. PLoS Pathog. 6:e1000873. doi: 10.1371/journal.ppat.1000873
Bruns, S., Seidler, M., Albrecht, D., Salvenmoser, S., Remme, N., Hertweck, C., et al. (2010b). Functional genomic profiling of Aspergillus fumigatus biofilm reveals enhanced production of the mycotoxin gliotoxin. Proteomics 10, 3097-3107. doi: 10.1002/pmic.201000129 acutely increases cystic fibrosis sputum elastase activity and its potential to induce lung hemorrhage in mice. Am. J. Respir. Crit. Care Med. 157, 464-469. doi: 10.1164/ajrccm.157.2.9608033

Chandra, J., Mukherjee, P. K., and Ghannoum, M. A. (2008). In vitro growth and analysis of Candida biofilms. Nat. Protoc. 3, 1909-1924. doi: 10.1038/nprot.2008.192

d'Enfert, C. (1996). Selection of multiple disruption events in Aspergillus fumigatus using the orotidine$5^{\prime}$-decarboxylase gene, $\operatorname{pyr} G$, as a unique transformation marker. Curr. Genet. 30, 76-82.

Da Silva Ferreira, M. E., Kress, M. R., Savoldi, M., Goldman, M. H., Hartl, A., Heinekamp, T., et al. (2006). The $a k u B$ (KU80) mutant deficient for nonhomologous end joining is a powerful tool for analyzing pathogenicity in Aspergillus fumigatus. Eukaryot. Cell. 5, 207-211. doi: 10.1128/EC.5.1.207-211.2006

Flemming, H. C., and Wingender, J. (2010). The biofilm matrix. Nat. Rev. Microbiol. 8, 623-633. doi: 10.1038/nrmicro2415

Fontaine, T., Beauvais, A., Loussert, C., Thevenard, B., Fulgsang, C. C., Ohno, N., et al. (2010). Cell wall alpha1-3glucans induce the aggregation of germinating conidia of Aspergillus fumigatus. Fungal Genet. Biol. 47, 707-712. doi: 10.1016/j.fgb.2010.04.006

Fuchs, T. A., Abed, U., Goosmann, C., Hurwitz, R., Schulze, I., Wahn, V., et al. (2007). Novel cell death program leads to neutrophil extracellular traps. J. Cell. Biol. 176, 231-241. doi: $10.1083 /$ jcb.200606027
Cantin, A. M. (1998). DNase I

development. It will be interesting to analyze whether eDNA originating from neutrophils, serves as a crucial nutrient source for the fungus and contributes to biofilm formation in vivo, especially in the DNA-rich mucus environment in the lungs of CF patients.

\section{ACKNOWLEDGMENTS}

This work was financially supported by the Hans Knöll Institute (HKI) and the Deutsche Forschungsgemeinschaft (DFG) funded excellence graduate school Jena School of Microbial communication (JSMC).

Gibbons, J. G., Beauvais, A., Beau, R., McGary, K. L., Latgé, J. P., and Rokas, A. (2012). Global transcriptome changes underlying colony growth in the opportunistic human pathogen Aspergillus fumigatus. Eukaryot. Cell. 11, 68-78. doi: 10.1128/EC.05102-11

Jöchl, C., Loh, E., Ploner, A., Haas, H., and Hüttenhofer, A. (2009). Development-dependent scavenging of nucleic acids in the filamentous fungus Aspergillus fumigatus. RNA Biol. 6, 179-186.

Latgé, J. P. (2001). The pathobiology of Aspergillus fumigatus. Trends Microbiol. 9, 382-389. doi: 10.1016/S0966-842X(01)02104-7

Lethem, M. I., James, S. L., Marriott, C. and Burke, J. F. (1990). The origin of DNA associated with mucus glycoproteins in cystic fibrosis sputum. Eur. Respir. J. 3, 19-23.

Loussert, C., Schmitt, C., Prevost, M. C., Balloy, V., Fadel, E., Philippe, B., et al. (2010). In vivo biofilm composition of Aspergillus fumigatus. Cell Microbiol. 12, 405-410. doi 10.1111/j.1462-5822.2009.01409.x

Mariscal, A., Lopez-Gigosos, R. M., Carnero-Varo, M., and FernandezCrehuet, J. (2009). Fluorescent assay based on resazurin for detection of activity of disinfectants against bacterial biofilm. Appl. Microbiol. Biotechnol. 82, 773-783. doi: 10.1007/s00253-0091879-x

Martins, M., Henriques, M., LopezRibot, J. L., and Oliveira, R. (2012). Addition of DNase improves the in vitro activity of antifungal drugs against Candida albicans biofilms. Mycoses 55, 80-85. doi: 10.1111/j.1439-0507.2011.02047.x

Martins, M., Uppuluri, P., Thomas, D. P., Cleary, I. A., Henriques, M., Lopez-Ribot, J. L., et al. (2010). Presence of extracellular DNA in the Candida albicans biofilm matrix and its contribution to biofilms. Mycopathologia 169, 323-331. doi: 10.1007/s11046-0099264-y
Mowat, E., Butcher, J., Lang, S., Williams, C., and Ramage, G. (2007). Development of a simple model for studying the effects of antifungal agents on multicellular communities of Aspergillus fumigatus. J. Med. Microbiol. 56, 1205-1212. doi: 10.1099/jmm.0.47247-0

Mowat, E., Williams, C., Jones, B., McChlery, S., and Ramage, G. (2009). The characteristics of Aspergillus fumigatus mycetoma development: is this a biofilm? Med. Mycol. 47(Suppl. 1), S120-S126. doi: 10.1080/13693780802238834

Mulcahy, H., Charron-Mazenod, L., and Lewenza, S. (2008). Extracellular DNA chelates cations and induces antibiotic resistance in Pseudomonas aeruginosa biofilms. PLoS Pathog. 4:e1000213. doi: 10.1371/journal.ppat.1000213

Muszkieta, L., Beauvais, A., Pahtz, V., Gibbons, J. G., Anton Leberre, V., Beau, R., et al. (2013). Investigation of Aspergillus fumigatus biofilm formation by various "omics" approaches. Front. Microbiol. 4:13. doi: 10.3389/fmicb.2013.00013

Nivoix, Y., Velten, M., Letscher-Bru, V., Moghaddam, A., NatarajanAme, S., Fohrer, C., et al. (2008). Factors associated with overall and attributable mortality in invasive aspergillosis. Clin. Infect. Dis. 47, 1176-1184. doi: 10.1086/592255

Papayannopoulos, V., Staab, D., and Zychlinsky, A. (2011). Neutrophil elastase enhances sputum solubilization in cystic fibrosis patients receiving DNase therapy. PLoS ONE 6:e28526. doi: 10.1371/journal.pone.0028526

Pierce, C. G., Uppuluri, P., Tristan, A. R., Wormley, F. L. Jr., Mowat, E., Ramage, G., et al. (2008). A simple and reproducible 96-well plate-based method for the formation of fungal biofilms and its application to antifungal susceptibility testing. Nat. Protoc. 3, 1494-1500. doi: 10.1038/nport. 2008.141 
Pihet, M., Carrere, J., Cimon, B., Chabasse, D., Delhaes, L., Symoens, F., et al. (2009). Occurrence and relevance of filamentous fungi in respiratory secretions of patients with cystic fibrosis-a review. Med. Mycol. 47, 387-397.

Rajendran, R., Williams, C., Lappin, D. F., Millington, O., Martins, M., and Ramage, G. (2013). Extracellular DNA release acts as an antifungal resistance mechanism in mature Aspergillus fumigatus biofilms. Eukaryot. Cell. 12, 420-429. doi: 10.1128/EC.00287-12

Ramage, G., Mowat, E., Jones, B., Williams, C., and Lopez-Ribot, J. (2009). Our current understanding of fungal biofilms. Crit. Rev. Microbiol. 35, 340-355. doi: 10.3109/10408410903241436

Sambrook, J., and Russell, D. W. (2001) Molecular cloning: A Laboratory Manual, 3rd Edn. Cold Spring Harbor, NY: Cold Spring Harbor Laboratory.

Scharf, D. H., Heinekamp, T., Remme, N., Hortschansky, P., Brakhage, A. A., and Hertweck, C. (2012). Biosynthesis and function of gliotoxin in Aspergillus fumigatus. Appl. Microbiol. Biotechnol. 93, 467-472. doi: 10.1007/s00253-011-3689-1
Seidler, M. J., Salvenmoser, S., and Muller, F. M. (2008). Aspergillus fumigatus forms biofilms with reduced antifungal drug susceptibility on bronchial epithelial cells. Antimicrob. Agents Chemother. 52, 4130-4136. doi: 10.1128/AAC.00234-08

Shak, S., Capon, D. J., Hellmiss, R., Marsters, S. A., and Baker, C. L. (1990). Recombinant human DNase I reduces the viscosity of cystic fibrosis sputum. Proc. Natl. Acad. Sci. U.S.A. 87, 9188-9192. doi: 10.1073/pnas.87. 23.9188

Spikes, S., Xu, R., Nguyen, C. K., Chamilos, G., Kontoyiannis, D. P., Jacobson, R. H., et al. (2008). Gliotoxin production in Aspergillus fumigatus contributes to hostspecific differences in virulence. J. Infect. Dis. 197, 479-486. doi: $10.1086 / 525044$

Tekaia, F., and Latgé, J. P. (2005). Aspergillus fumigatus: saprophyte or pathogen? Curr. Opin. Microbiol. 8, 385-392. doi: 10.1016/j.mib.2005.06.017

Toyotome, T., Yamaguchi, M., Iwasaki, A., Watanabe, A., Taguchi, H., Qin, L., et al. (2012). Fetuin A, a serum component, promotes growth and biofilm formation by Aspergillus fumigatus. Int. J. Med. Microbiol. 302, 108-116.

Urban, C. F., Ermert, D., Schmid, M., Abu-Abed, U., Goosmann, C., Nacken, W., et al. (2009) Neutrophil extracellular traps contain calprotectin, a cytosolic protein complex involved in host defense against Candida albicans. PLoS Pathog. 5:e1000639. doi: 10.1371/journal.ppat.1000639

Weidner, G., D'enfert, C., Koch, A., Mol, P. C., and Brakhage, A. A. (1998). Development of a homologous transformation system for the human pathogenic fungus Aspergillus fumigatus based on the pyrG gene encoding orotidine $5^{\prime}$-monophosphate decarboxylase. Curr. Genet. 33, 378-385.

Whitchurch, C. B., Tolker-Nielsen, T., Ragas, P. C., and Mattick, J. S. (2002). Extracellular DNA required for bacterial biofilm formation. Science 295, 1487. doi: 10.1126/science.295.5559.1487

Yang, Y., Sreenivasan, P. K., Subramanyam, R., and Cummins, D. (2006). Multiparameter assessments to determine the effects of sugars and antimicrobials on a polymicrobial oral biofilm. Appl. Environ. Microbiol. 72,
6734-6742. doi: 10.1128/AEM. 01013-06

Conflict of Interest Statement: The authors declare that the research was conducted in the absence of any commercial or financial relationships that could be construed as a potential conflict of interest.

Received: 15 March 2013; accepted: 17 May 2013; published online: 06 June 2013.

Citation: Shopova I, Bruns S, Thywissen $A$, Kniemeyer $O$, Brakhage $A A$ and Hillmann $F$ (2013) Extrinsic extracellular DNA leads to biofilm formation and colocalizes with matrix polysaccharides in the human pathogenic fungus Aspergillus fumigatus. Front. Microbiol. 4:141. doi: 10.3389/fmicb.2013.00141

This article was submitted to Frontiers in Fungi and Their Interactions, a specialty of Frontiers in Microbiology.

Copyright (c) 2013 Shopova, Bruns, Thywissen, Kniemeyer, Brakhage and Hillmann. This is an open-access article distributed under the terms of the Creative Commons Attribution License, which permits use, distribution and reproduction in other forums, provided the original authors and source are credited and subject to any copyright notices concerning any third-party graphics etc. 